\title{
Bilateral posterior cerebral artery stroke following transtentorial herniation caused by a subependymal giant cell astrocytoma in a patient with tuberous sclerosis: a case report
}

Joong-Goo Kim, MD'; Jay Chol Choi, MD, PhD'; Hong Jun Kim, MD; Jong Kook Rhim, MD² Tae Jun Jung ${ }^{3}$; Chang-Lim Hyun, MD, PhD; Jin Deok Joo, MD²

\author{
'Department of Neurology, Jeju National University Hospital, Jeju National University School of \\ Medicine, Jeju, Korea \\ ${ }^{2}$ Department of Neurosurgery, Jeju National University Hospital, Jeju National University School of \\ Medicine, Jeju, Korea \\ ${ }^{3}$ Jeju National University School of Medicine, Jeju, Korea \\ ${ }^{4}$ Department of Pathology, Jeju National University School of Medicine, Korea
}

\section{Journal of \\ Neurocritical \\ Care \\ CASE REPORT \\ Received: November 10, 2021 \\ Revised: December 5, 2021 \\ Accepted: December 6, 2021 \\ Corresponding Author: \\ Jin Deok Joo, MD \\ Department of Neurosurgery, Jeju \\ National University Hospital, Jeju \\ National University School of Medicine, \\ 15 Aran 13-gil, Jeju 63241, Korea \\ Tel: +82-64-717-1620 \\ Fax: +82-64-717-1630 \\ E-mail: etude9@gmail.com}

Background: Acute increased intracranial pressure (IICP) is a life-threatening condition that requires urgent treatment. Rapid IICP with hydrocephalus may be complicated by ischemic stroke, convulsions, loss of consciousness, brain herniation, and death. Extremely rare complications include intracranial vessel entrapment and ischemic stroke due to sudden IICP in cases with benign tumors.

Case Report: We report a case of bilateral posterior cerebral artery region infarction and complicated hydrocephalus with subependymal giant cell astrocytoma in a patient with tuberous sclerosis.

Conclusion: We postulate that the temporary IICP induced by seizure led to transient bilateral posterior cerebral artery entrapment, causing ischemic stroke without vascular occlusion.

Keywords: Tuberous sclerosis; Astrocytoma; Stroke; Intracranial pressure; Hydrocephalus

\section{INTRODUCTION}

Tuberous sclerosis complex (TSC) is an autosomal dominant multisystem disorder, first described by Bourneville [1]. TSC patients present with three distinct intracranial lesions: subcortical tubers, subependymal nodules, and subependymal giant cell astrocytomas (SEGA) [1,2]. Despite its benign histopathology, SEGA can result in a variety of severe events that may substantially increase the mortality and morbidity of TSC patients [3]. This is due to their preferential proximity to the foramen of Monro, which can get blocked during tumor growth, causing obstructive hydrocephalus [4]. Obstructive hydrocephalus can be complicated by convulsions, loss of consciousness, brain herniation, and death [5].

Usually, SEGA grows very slowly, rarely inducing acute increased intracranial pressure (IICP), which can be life threatening [6]. Intracranial vessel compression induced by IICP in SEGA patients has not been reported. We report a rare case of acute bilateral occipital lobe infarction due to SEGA presenting with acute obstructive hydrocephalus. 


\section{CASE REPORT}

A 33-year-old man was brought to the emergency room (ER) with altered mental status. He had a history of recurrent seizures and mental retardation related to TSC first diagnosed at the age of 17 years, when contrast-enhanced T1-weighted imaging revealed a $1.4 \times 0.9-\mathrm{cm}^{2}$ intraventricular tumor (Fig. 1A). The patient visit- ed the hospital frequently with generalized seizures and changes in mental status. At the age of 31 years, he developed decreased appetite, fatigue, somnolence, and projectile vomiting. T1-enhanced images revealed a $4 \times 6.1 \times 5.5-\mathrm{cm}^{3}$ well-defined mass in the midline of the lateral ventricle (Fig. 1B). Fluid-attenuated inversion recovery imaging revealed marked ventricular dilatation with a high-signal halo around the lateral ventricles, suggesting
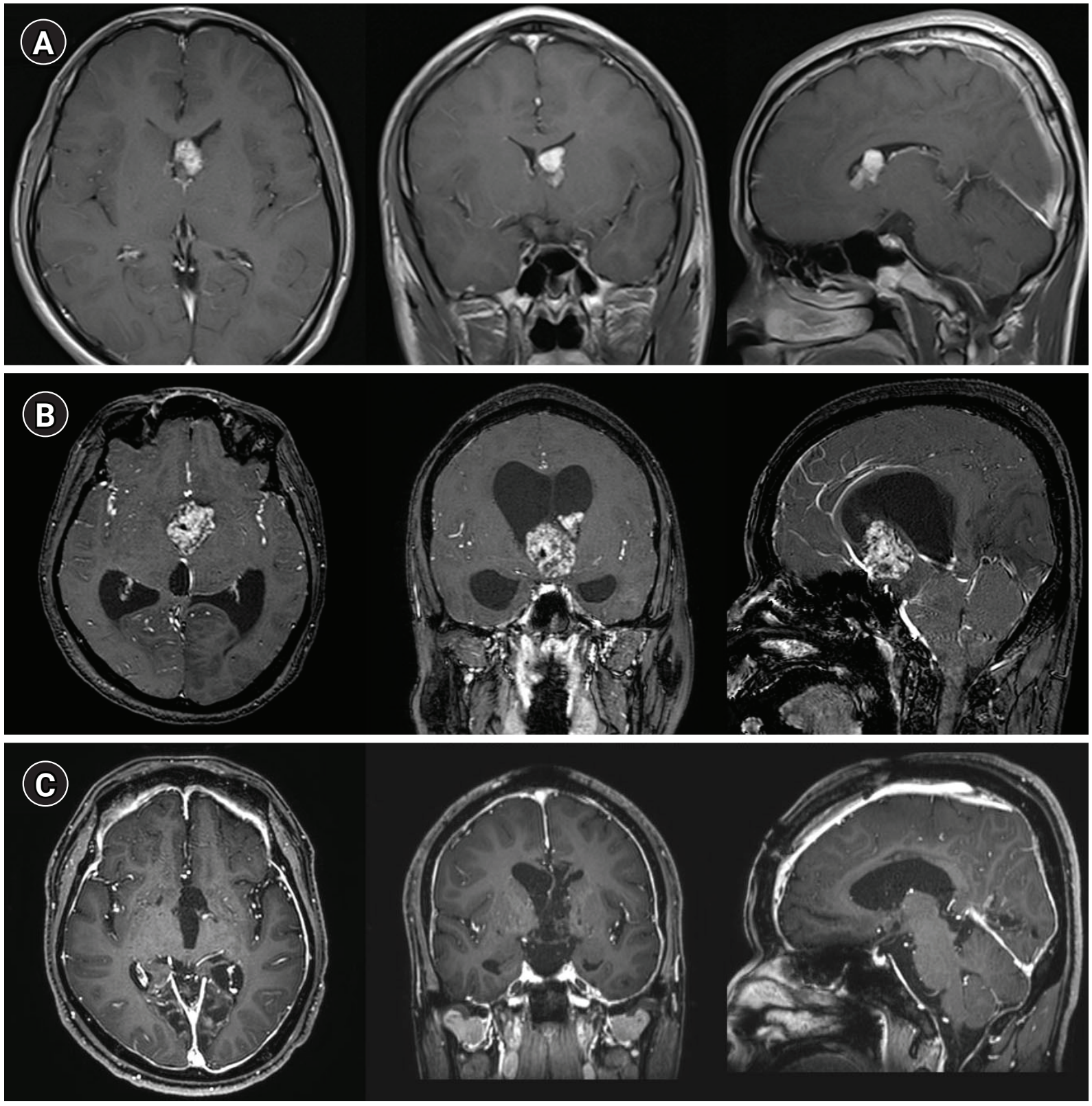

Fig. 1. (A) The axial/coronal/sagittal contrast-enhanced T1-weighted sequence from 16 years earlier showed a solid homogeneous lesion in the left lateral ventricle, extending toward the foramen of Monro without hydrocephalus. (B) Immediately preoperatively, the contrast-enhanced T1-weighted sequence showed marked heterogeneous enhancement and proximal aqueduct occlusion with marked hydrocephalus. Coronal and sagittal magnetic resonance imaging images showed marked dilatation of the lateral and third ventricles, with a normal-sized fourth ventricle. Note the downward bowing of the third ventricle floor with expanded infundibular and optic recesses. (C) Postoperative contrast-enhanced T1 axial scans showed no residual tumor with marked meningeal enhancement. 
hydrocephalus. Marked growth of the ventricular lesion was noted.

The patient's symptoms usually improved after seizure control. This time, however, there was failure of recovery of consciousness after monitoring in the ER for 3 hours. At the time of admission, his blood pressure was slightly elevated at $150 / 90 \mathrm{mmHg}$, but other vital signs were normal. A neurological examination revealed that the patient was stuporous and unable to open his eyes on command. There was no apparent limb weakness and he showed an avoidance response to painful stimuli. Both pupils were $5 \mathrm{~mm}$ in diameter and showed decreased light reactivity. Direct ophthalmoscopy suggested papilledema with no evidence of disc cupping or atrophic changes. Diffusion-weighted imaging showed diffusion restriction of both occipital lobes suggesting acute ischemic stroke (Fig. 2A). Digital subtraction angiography showed maintained patency and normal caliber of both posterior cerebral arteries (PCAs) without significant perfusion defect (Fig. 2B).

The patient was diagnosed with bilateral occipital lobe infarction due to obstructive hydrocephalus and referred to neurosurgery. First, a ventriculoperitoneal shunt was inserted in the ER to relieve the IICP. The patient underwent a bifrontal craniotomy, and the tumor was exposed via an interhemispheric transchoroidal approach. The mass originated from the anterior wall of the third ventricle and extruded into the left lateral ventricle via the foramen of Monro. The mass was yellow to pinkish with moderate vascularity. Gross total resection was achieved after identifying the opening of the cerebral aqueduct.

Histological evaluation of the mass led to the diagnosis of
SEGA. Hematoxylin and eosin staining showed large polygonal cells resembling astrocytes or ganglion cells. The tumor cells had abundant, finely granular eosinophilic cytoplasm with large round to oval nuclei and prominent nucleoli (Fig. 3A and B). Immunohistochemistry was positive for S-100 and glial fibrillary acidic protein (Fig. 3C and D). A 12-lead electrocardiogram, transthoracic echocardiogram, and heart rhythm monitor during the intensive care unit admission were normal. There was no significant evidence of hypercoagulable state in the initial young age stroke evaluation. On discharge from the hospital, the patient had recovered all symptoms except for cortical blindness. One year postoperatively, magnetic resonance imaging (MRI) showed complete resection of the SEGA and resolution of the hydrocephalus (Fig. 1C). However, the visually evoked potential was still absent in both eyes. Despite patency of the PCAs, the visual loss did not show recovery.

\section{DISCUSSION}

SEGA is a low-grade astrocytoma that arises within the cerebral ventricles in patients with TSC [3]. Although SEGA can occur in the absence of TSC, it quite frequent in TSC, occurring in 5\%$20 \%$ of TSC cases [2]. It is generally benign, as the lesions grow slowly and rarely infiltrate the adjacent brain tissue [4]. However, in case of uncontrolled hydrocephalus, TSC may lead to progressive obstructive hydrocephalus and transtentorial herniation [7]. Although the related symptoms are usually mild and can be relieved by emergency shunt insertion, acute IICP can be fatal [6].

The distinguishing feature of our case was that the flow in both
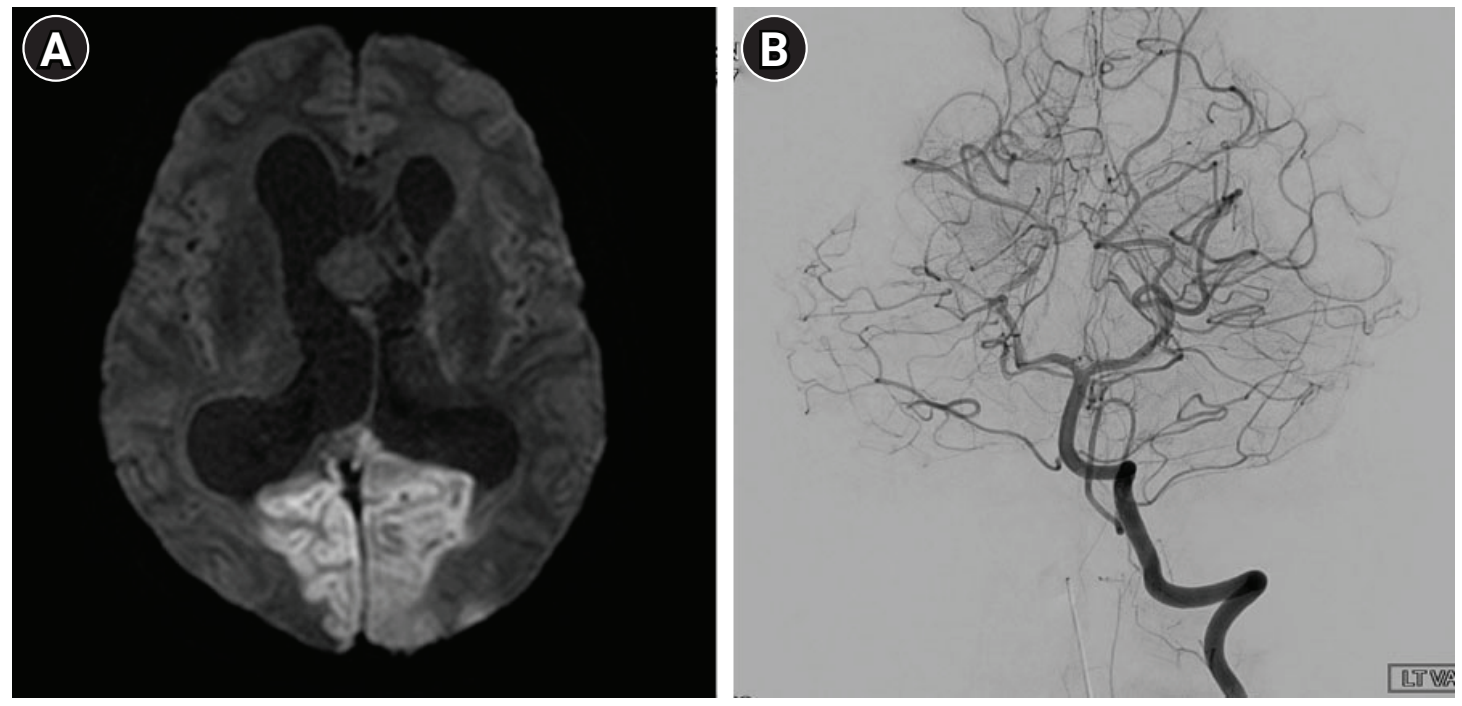

Fig. 2. (A) Diffusion-weighted image showed hyperintensity suggesting acute ischemic stroke in the region of the bilateral posterior cerebral artery (PCA). (B) Conventional angiography showed patency of the lumens of both PCAs, which have normal diameters. 

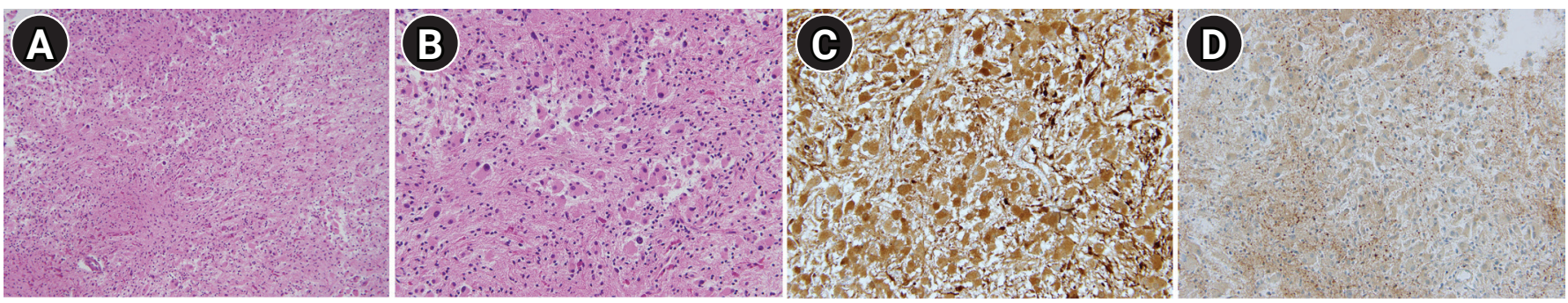

Fig. 3. (A, B) Microscopically, the tumor comprised mainly of large polygonal cells resembling astrocytes or ganglion cells. The tumor cells have abundant, finely granular eosinophilic cytoplasm with large round to oval nuclei and prominent nucleoli $\left(H \& E_{1}, A: \times 100, B: \times 200\right)$. (C, D) Tumor cells show positive immunoreactivity for S-100 and glial fibrillary acidic protein (immunohistochemical stain, $\times 200$ ).

PCAs was maintained after an irreversible ischemic stroke. Considering the growth rate of SEGA, the entrapment or compression of intracranial vessels by transtentorial herniation is extremely rare. This suggests that other factors contributed to the temporary deterioration following IICP. Recurrent seizures are common in patients with TSC, with a reported prevalence of $62 \%-93 \%$ [4]. Excessive IICP in TSC patients is thought to cause and exacerbate seizure attacks, and several studies have suggested that seizures can lead to transient rapid IICP [8].

Therefore, it is theoretically possible that seizures and IICP may exacerbate each other. The cerebral infarction in both PCAs without definite arterial occlusion on conventional angiography suggests a temporary interruption during the instantaneous IICP associated with seizure. Consequently, the interaction between the bilateral rostrocaudal descent and horizontal midline shift led to compression of both PCAs [7].

The enlarged lateral ventricle independently worsened the compartment pressure via the "accordion" effect [9]. The combined forces associated with the midline shift and ventricular enlargement resulted in descent of the medial temporal lobe, causing impingement of the PCA on the rigid tentorial edge. In this process, acute IICP in hydrocephalus most often involves the PCAs [9]. Considering the anatomical course of the PCAs as they pass back up over the medial edge of the tentorium, ischemic stroke related to hydrocephalus may develop in the PCA region rather than the anterior circulation [10]. The cause of extension of the infarcted lesion limited medial occipital lobe was probably associated with collateral flows of anterior circulation and, the direction of PCAs. More medially developed PCAs have been presumed to affect the medial occipital lobe during arterial entrapment.

No case of hydrocephalus and IICP caused by SEGA leading to ischemic stroke has been reported previously. The differentiation of epilepsy from an IICP crisis is not always obvious, but is mandatory. Careful neurological examination and MRI, including diffusion imaging and gadolinium enhancement, are crucial examinations during differential diagnosis, and both should be includ- ed in the diagnostic protocol of stroke with the slightest sign of brain tumor. Patients presenting with TSC with SEGA causing obstructive hydrocephalus is an extremely rare cause of ischemic stroke associated with malignant IICP.

\section{ARTICLE INFORMATION}

\section{Ethics statement}

Ethics approval from Institutional Review Board of Jeju National University Hospital was granted in accordance with national requirements (IRB No. 2021-12-011), and the need for written informed from a patient was waived.

\section{Conflict of interest}

No potential conflict of interest is relevant to this article.

\section{Funding}

This work was supported by a 2019 research grant from Jeju National University Hospital.

\section{ORCID}

Joong-Goo Kim https://orcid.org/0000-0002-4166-6023

Jay Chol Choi https://orcid.org/0000-0002-3550-2196

Hong Jun Kim https://orcid.org/0000-0003-2623-5951

Jong Kook Rhim https://orcid.org/0000-0003-2538-8374

Tae Jun Jung https://orcid.org/0000-0001-5051-1261

Chang-Lim Hyun https://orcid.org/0000-0002-6740-1357

Jin Deok Joo https://orcid.org/0000-0002-0728-3336

\section{Author contributions}

Conceptualization: JGK, JDJ. Data curation: HJK, TJJ. Formal analysis: JKR, JGK, JDJ, CLH. Funding acquisition: JDJ. Methodology: JCC. Project administration: JGK. Visualization: JGK, JDJ. Writing-original draft: JGK. Writing-review \& editing: all authors. 


\section{REFERENCES}

1. Henske EP, Jóźwiak S, Kingswood JC, Sampson JR, Thiele EA. Tuberous sclerosis complex. Nat Rev Dis Primers 2016;2: 16035.

2. Crino PB, Nathanson KL, Henske EP. The tuberous sclerosis complex. N Engl J Med 2006;355:1345-56.

3. Roth J, Roach ES, Bartels U, Jóźwiak S, Koenig MK, Weiner $\mathrm{HL}$, et al. Subependymal giant cell astrocytoma: diagnosis, screening, and treatment. Recommendations from the International Tuberous Sclerosis Complex Consensus Conference 2012. Pediatr Neurol 2013;49:439-44.

4. Jansen AC, Belousova E, Benedik MP, Carter T, Cottin V, Curatolo $\mathrm{P}$, et al. Clinical characteristics of subependymal giant cell astrocytoma in tuberous sclerosis complex. Front Neurol 2019;10:705.

5. Von Haken MS, Aschoff AA, Diringer MN. Acute obstructive hydrocephalus. In: Hacke W, Hanley DF, Einhäupl KM, Bleck
TP, Diringer MN, Ropper AH, editors. Neurocritical care. Berlin, Heidelberg: Springer Berlin Heidelberg; 1994. p. 869-82.

6. Jóźwiak S, Nabbout R, Curatolo P, Participants of the TSC Consensus Meeting for SEGA and Epilepsy Management. Management of subependymal giant cell astrocytoma (SEGA) associated with tuberous sclerosis complex (TSC): clinical recommendations. Eur J Paediatr Neurol 2013;17:348-52.

7. Sgouros S, Malluci C, Walsh AR, Hockley AD. Long-term complications of hydrocephalus. Pediatr Neurosurg 1995;23:12732.

8. Gabor AJ, Brooks AG, Scobey RP, Parsons GH. Intracranial pressure during epileptic seizures. Electroencephalogr Clin Neurophysiol 1984;57:497-506.

9. Ansari M, Chang GY. Pathophysiology of stroke in the contralateral posterior cerebral artery distribution from a tentorial herniation. J Clin Neurol 2017;13:101-2.

10. Kuybu O, Tadi P, Dossani RH. Posterior cerebral artery stroke. Treasure Island, FL: StatPearls Publishing; 2021. 